Research Article

\title{
Miniaturized Spiral Metamaterial Array for a Ventilated Broadband Acoustic Absorber
}

\author{
Xingxing Liu $(\mathbb{D}$, Xiang Li, and Zhiying Ren $\mathbb{C}$ \\ School of Mechanical Engineering and Automation, Fuzhou University, Fuzhou, China \\ Correspondence should be addressed to Zhiying Ren; renzyrose@126.com
}

Received 17 September 2020; Revised 19 October 2020; Accepted 22 October 2020; Published 2 November 2020

Academic Editor: Kui Wang

Copyright (C) 2020 Xingxing Liu et al. This is an open access article distributed under the Creative Commons Attribution License, which permits unrestricted use, distribution, and reproduction in any medium, provided the original work is properly cited.

\begin{abstract}
The high-efficiency and broadband acoustic reduction performance in a miniaturized free-flow structure remains challenging in acoustic engineering applications due to the contradiction of ventilation and acoustic reduction performance. Traditional acoustic absorbers can sufficiently attenuate acoustic wave, but meanwhile, block fluid flow due to the longitudinal nature of acoustic waves that can transmit among any small holes. Although different types of ventilated metamaterial absorbers (VMAs) with proper reduction and ventilation performance have been demonstrated recently, their insufficiencies lie in small open-area ratios, not efficient-enough reduction performance, bulky structure, and narrow working band. To further solve existing defects, a ventilated broadband absorber with the miniaturized metamaterial structure has been proposed. The designed absorber consists of miniaturized Archimedean spiral units, which can be easily stacked to achieve broadband and ventilated performance. This work opens up possibilities for practical acoustic applications where both noise control and ventilation are required, especially in a small space.
\end{abstract}

\section{Introduction}

In the last decade, many types of acoustic metamaterial absorbers have been designed to solve intrinsic problems of traditional acoustic reduction materials when dealing with broadband acoustics, especially for low-frequency range [1-4]. Compared with traditional porous materials, special design of metamaterials can give rise to the high-efficiency noise reduction in flexible working frequencies [5-10]. They often own customized structures and can be mounted on narrow and humid spaces to control the noise for the requirement of sound-silent environment. However, in daily life and industry products, noise usually comes with the background fluid flow, especially for the turbulent flow in or around nozzles, ducts, turbines, and air conditioners [11-14]. The requirement of the free-flow pass makes many previous metamaterial absorbers incompetent, as they only work adequately in sealed conditions, while sound can penetrate very small holes [1-4]. Otherwise, the existence of free-pass holes or channels will drastically degrade the acoustic reduction performance [15-17].

Recently, several types of VMAs have been proposed and well analyzed [17-26]. However, their reduction and ventilation performances are still not so satisfying in real application scenarios, as few of them can balance different requirements such as noise reduction, fluid ventilation, limited mounted spaces, and broadband working ranges. Specifically, different types of designs and their related insufficiencies have been roughly classified into three categories, which are listed as follows. Some researchers $[18,25]$ used coupled Helmholtz resonators array and achieved good performance at high frequencies, which were also confirmed experimentally. Some researchers $[17,19,21]$ employed more complex coupling membrane resonators or rainbow-trapping resonators and achieved good acoustic reduction performance, but only have small open-area ratios, which did not display good ventilation performance. Other researchers $[20,24]$ designed the hollow pipe array perforated with deepsubwavelength microperforations or hybrid membrane resonators, and they all owned good ventilation performance but without efficient-enough acoustic reduction performance, especially in a broadband range. In contrast, some novel ventilated acoustic barriers [27-33] did achieve high-efficiency ventilation ( $>60 \%$ wind velocity ratio) and acoustic reflection $(>90 \%)$ at relatively low frequencies, but meanwhile, still cannot avoid disadvantages of narrow working frequencies and relative bulky structures. The performance 
deficiency for existing VMAs can attribute to some reasons, such as the strict trade-off between ventilation and acoustic reduction performance and the intrinsic capabilities effective for subwavelength scatters [34], especially in low frequencies. Accordingly, being able to break existing limitations and achieve a miniaturized broadband absorber with good ventilation and acoustic reduction performance plays a key role in many acoustic application scenarios in noise control of nozzles, ducts, turbines, and air conditioners, which are commonly used in automobiles and buildings.

In this work, a ventilated broadband acoustic absorber with miniaturized spiral-type metamaterial lattice array is proposed. The metamaterial lattice array consists of weakly coupled Archimedean spiral resonators, and different unit combinations have been analyzed and compared to achieve the optimized ventilation and acoustic reduction performance. The resulted optimized stacking of the designed metamaterial is numerically and experimentally demonstrated. The extralong inner waveguides formed by Archimedean spiral configurations improve the targeted performance and own a wide working frequency range in a relatively miniaturized structure (the width of one single unit can be around $10 \mathrm{~mm}$ ). The complex spiral-generated long waveguide provides a new diagram for designing effective VMAs. This design steps further in practical applications for the noise control in an environment covered with flowing fluids such as flow ducts, exhaust hoods, and air conditioners.

\section{Prototype Design and Principal Explanation}

As it is shown in Figure 1, the designed array of VMA units is assembled by three Archimedean spiral units with different combinations. Figures 1(b) and 1(e) are the 3D prototype model, while Figures 1(a) and 1(d) are corresponding 3Dprinted products, which are fabricated by epoxy resins and are adjusted to be available for testing in the impedance tube (more details can be found in Supplementary Materials). The large gap between up-down Archimedean spiral units in the frame permits fluid flows easily. Moreover, the designed VMA unit does not need to be packaged in a specific Helmholtz resonator or a rectangular structure, which means it is flexible to mount in any needed devices or structures to realize the requested ventilation and acoustic reduction performance. In this work, the structure is assumed immersing in air. Figures 1(c) and 1(f) show the horizontal cross section of the metamaterial array, which consists of three VMA units. The length $L=35 \mathrm{~mm}$ and thickness $h_{1}=0.5 \mathrm{~mm}, h_{4}=0.75 \mathrm{~mm}$, and $h_{5}=15 \mathrm{~mm}$ are considered as fixed values, while the distance values of $h_{2}$ and $h_{3}$ are considered as adjustable geometric parameters. The governing equation of the Archimedean spiral is described as $x=(0.2 \theta+0.1) \cos (\theta)$ $(\mathrm{mm})$ and $y=(0.2 \theta+0.1) \sin (\theta)(\mathrm{mm})$. In Figure $1(\mathrm{c})$, ending sweeping points are denoted as $\theta_{1}=3.25 \times 2 \pi$, $\theta_{2}=4.25 \times 2 \pi$, and $\theta_{3}=5.25 \times 2 \pi$ for the corresponding left spiral, middle spiral, and right spiral, respectively. All uniform spiral unit arrays in the form of Figure 1(f) in the following paragraphs are using the same size of the left spiral in Figure 1(c). This work will also optimize the self-segmentation array (shown in Supplementary Materials), the spiral rotation direction, and the distance between adjacent spirals to realize the broadband and miniaturized VMA.

To investigate the effect of different optimized methods on the acoustic reduction performance of the designed VMA, fullwave numerical simulations are performed using COMSOL Multiphysics (detailed setup can be seen in Supplementary Materials). Here, the acoustic reduction coefficient $(R)$ is defined as $R=1-|T c|$, where $T c$ is defined as the transmission coefficient. Note that both $R$ and $T c$ are plotted in terms of amplitude and not in terms of acoustic energy in the following figures, in order to clearly compare the curve of the acoustic reduction performance. As shown in Figure 2(a), the value of $R$ as the change of frequency for the rectangular channel with one Archimedean spiral unit is plotted, and the inset shows the acoustic pressure distribution in the peak frequency. The width of the unit can be around $10 \mathrm{~mm}$, while the first resonant frequency is $1316 \mathrm{~Hz}$, which means the wavelength is 26 times larger than the characteristic dimension of the unit. Also, in Figure 2(a), we can see that the acoustic energy is strongly localized in this deep-subwavelength unit, as the effective reduction frequency range $(R>0.5)$ can be around $50 \mathrm{~Hz}$. The same Archimedean spiral unit array and the reversed array are shown in Figure 2(b), we can barely tell the acoustic reduction coefficient difference between the same array and the middlereversed array, which means the rotation direction will not affect the reduction performance in this type of VMAs. The major part of the acoustic energy is locked in the first unit, and the related effective frequency range can be around $160 \mathrm{~Hz}$. That means, even one single spiral unit can achieve good acoustic reduction performance and remains good ventilation performance as the gap between the boundary and the edge of the unit is big enough for fluid flows.

\section{Effects of Spiral Unit Combinations and Theoretical Analysis}

Figure 3 shows the optimization of distances between adjacent spirals, to realize the broadband and miniaturized VMA. Figure 3(a) displays different combinations of distances $h_{2}$ and $h_{3}$, and all the simulation results are compared with the experimental data of the combination of " $9.75-22 \mathrm{~mm}$ " array, whose specific configuration and dimensions are shown in Figure 1(c). As shown in Figure 3(a), we can clearly see the combination of " $9.75-22 \mathrm{~mm}$ " array displaying the best acoustic reduction performance, which is also verified in the experiment, as the experimental data fit well with the simulation data of this array. Moreover, as shown in Figure 3(b), for the related structure, all pressure distributions of four peak frequency points are displayed. In $851 \mathrm{~Hz}$, the acoustic energy is localized in the third spiral unit, as the acoustic source is placed on the left side, and the corresponding effective reduction frequency range lies in $840-875 \mathrm{~Hz}$, which demonstrates a good low-frequency reduction performance in this deep-subwavelength array. In $1316 \mathrm{~Hz}$, the acoustic energy is localized in the second spiral unit, the corresponding effective reduction frequency range lies in $1291-1403 \mathrm{~Hz}$, and meanwhile, due to the existence of weak coupling between the second and third spiral unit, some degrees of acoustic reduction ability are shown among the first two peak frequencies. In $2292 \mathrm{~Hz}$, the acoustic 


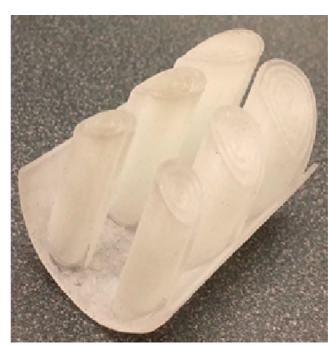

(a)

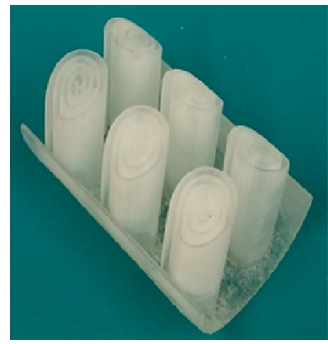

(d)

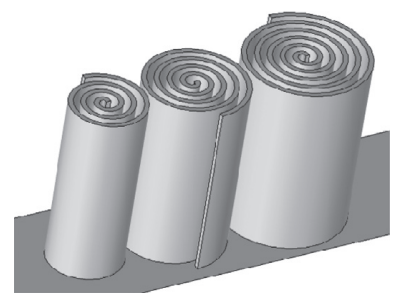

(b)

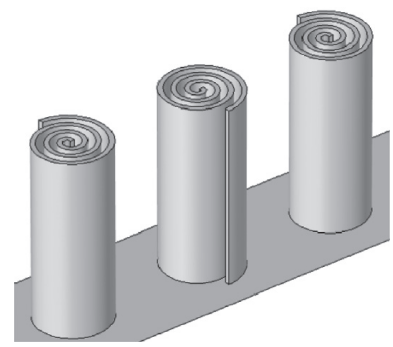

(e)

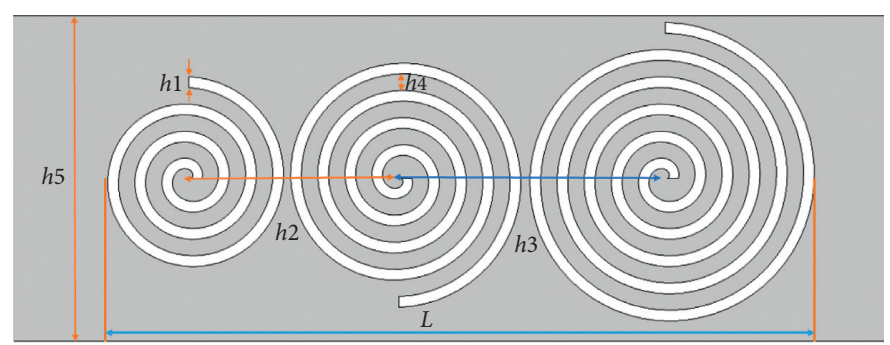

(c)

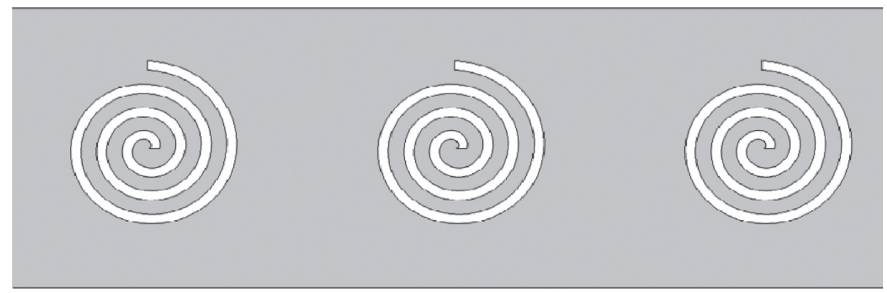

(f)

Figure 1: (a) The 3D-printed product for the varied-unit spiral array. (b) The corresponding 3D prototype model of (a). (c) The horizontal cross section and detailed dimensions of (a). (d) The 3D-printed product for the same spiral unit array. (e) The corresponding 3D prototype model of (d) but with the middle spiral reversed. (f) The horizontal cross section of (e).

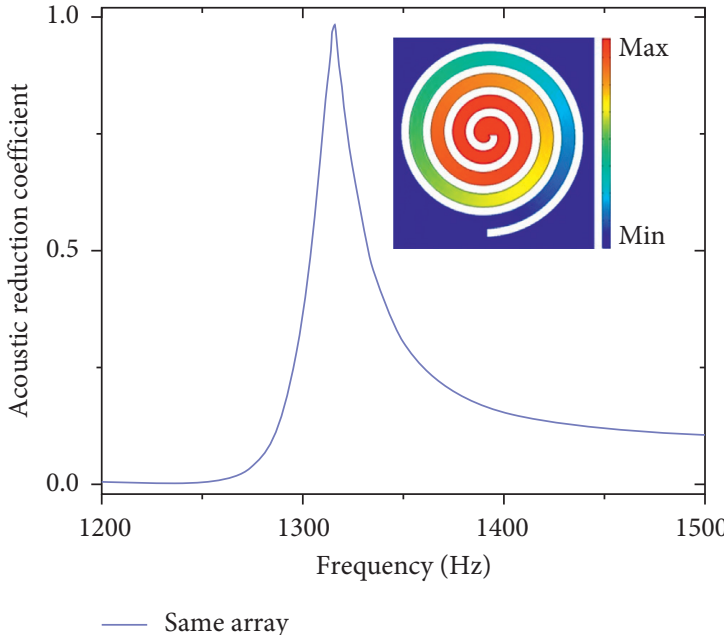

(a)

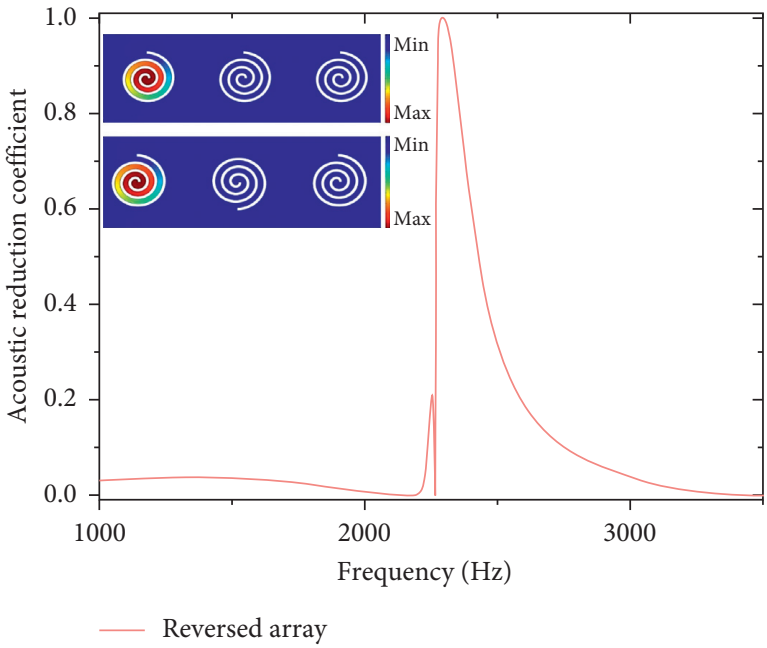

(b)

FIgURE 2: (a) The acoustic reduction coefficient for the rectangular channel with one Archimedean spiral unit. The inset shows the acoustic pressure distribution in the peak frequency. (b) The comparison of the same spiral unit array and the middle-reversed array for the acoustic reduction coefficient. The insets show the corresponding acoustic pressure distributions in peak frequencies.

energy is localized in the first spiral unit, the corresponding effective reduction frequency range lies in $2190-2366 \mathrm{~Hz}$, and due to the existence of weak coupling between the first and second spiral unit, relative good acoustic reduction performance is shown among the second and third peak frequencies. In $2554 \mathrm{~Hz}$, the acoustic energy is localized in the first and third spiral units, and the corresponding effective reduction frequency range lies in $2525-2808 \mathrm{~Hz}$. Therefore, the combination of "9.75-22 mm" varied-unit array displays the best acoustic reduction performance and can be considered a broadband miniaturized VMA.
The theoretical description is performed by the transfer matrix method (TMM) $[35,36]$, which includes the acoustic pressure $p_{i}$, acoustic particle velocity $u_{i}$, and the transfer matrix $T_{i}$. For simplicity, in this design, it is assumed that the metamaterial array contains an $i$-layer self-segmentation spiral unit, and the space occupied by each unit is set as $a_{i}$. Then, we can establish the transfer matrix between two adjacent spiral unit spaces as $\left[p_{i} u_{i}\right]=\left[p_{i+1} u_{i+1}\right]\left[T_{i}\right]^{\mathrm{T}}$, in which the position of $p_{i}$ and $u_{i}$ for each unit space is indicated by $m$ on the left and $n$ on the right, as shown in Figure 1(b). When acoustic waves transmit in the 


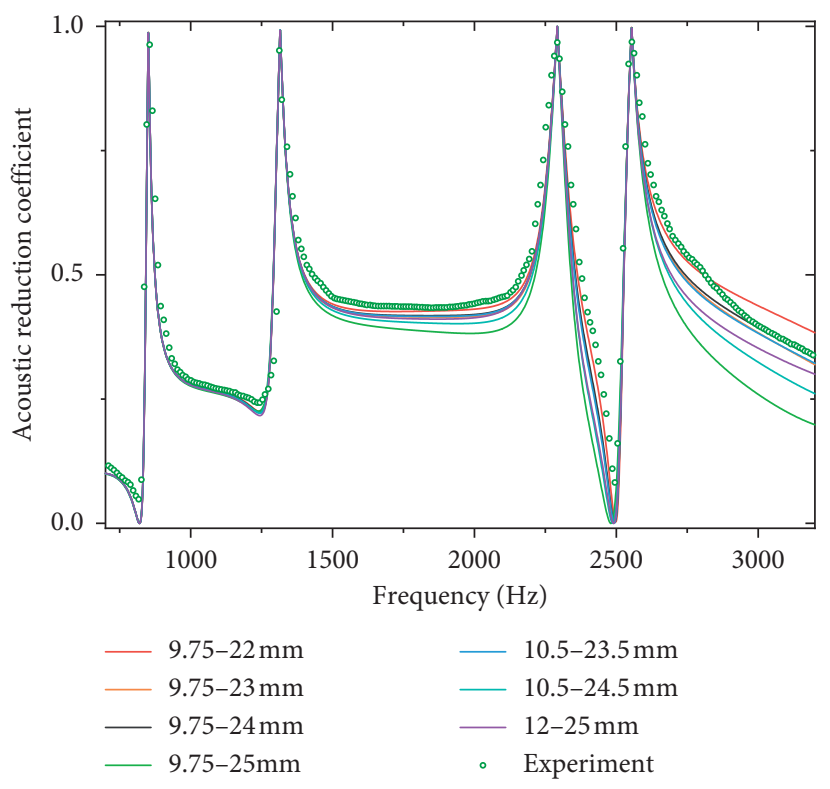

(a)

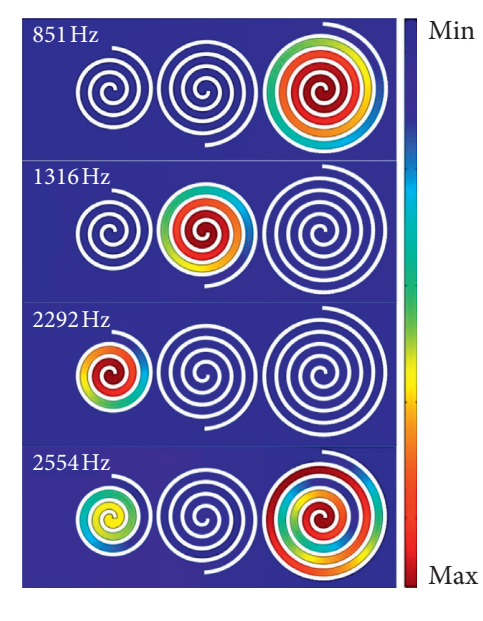

(b)

Figure 3: (a) Different simulation results for spiral unit combinations of distances $h_{2}$ and $h_{3}$ and comparison with the experimental data for the combination of " $9.75-22 \mathrm{~mm}$ " array. (b) Pressure distributions of four peak frequency points.

Archimedean spiral array channel, the thermoviscous effect should be considered in the theoretical analysis and the numerical simulation process, due to the existence of the viscosity in tiny structures. The thermoviscous effect can be characterized by the complex wave number $k_{\text {eff, } i}$ and the effective impedance $Z_{\mathrm{eff}, i}$. So, the general expression can be written as

$$
\left[T_{i}\right]=\left[\begin{array}{cc}
\cos \left(k_{\mathrm{eff}, i} a_{i}\right) & j Z_{\mathrm{eff}, i} \sin \left(k_{\mathrm{eff}, i} a_{i}\right) \\
\frac{j \sin \left(k_{\mathrm{eff}, i} a_{i}\right)}{Z_{\mathrm{eff}, i}} & \cos \left(k_{\mathrm{eff}, i} a_{i}\right)
\end{array}\right] .
$$

The transfer matrix $\left[T_{i}\right]$ can be solved by using results of COMSOL Multiphysics, as the existence of the varied-unit complex spiral structure. All details are shown in Figure 4(a), and periodic boundary conditions are applied to two edges of the channel. To further investigate this theoretical model, we can take the $i$ th spiral unit out of this metamaterial system independently and apply sinusoidal thermoviscous acoustic pressure signal. Assuming pressures $\left(p_{m}, p_{n}\right)$ and velocities $\left(u_{m}, u_{n}\right)$ can be obtained from simulation results, all the corresponding variables can be written in the form of $p_{m}, p_{n}, u_{m}$ and $u_{n}$, and finally we have

$$
\left[T_{i}\right]=\left[\begin{array}{cc}
\frac{p_{n} u_{n}+p_{m} u_{m}}{p_{m} u_{n}+p_{n} u_{m}} & \mathrm{Z}_{\mathrm{eff}, i}^{2} \frac{p_{m} u_{n}-p_{n} u_{m}}{-p_{n}^{2}+u_{n}^{2} Z_{\mathrm{eff}, i}^{2}} \\
\frac{p_{m} u_{n}-p_{n} u_{m}}{-p_{n}^{2}+u_{n}^{2} Z_{\mathrm{eff}, i}^{2}} & \frac{p_{n} u_{n}+p_{m} u_{m}}{p_{m} u_{n}+p_{n} u_{m}}
\end{array}\right],
$$

where $Z_{\text {eff }, i}^{2}=\left(p_{m}^{2}-p_{n}^{2}\right) /\left(u_{m}^{2}-u_{n}^{2}\right)$. The transfer matrix for every unit space can be determined in such a similar method, so the overall transfer matrix can be written as $[\mathbf{T}]=\left[T_{1}\right]\left[T_{2}\right]$ $\left[T_{3}\right], \ldots \ldots . .,\left[T_{i}\right]$. Here, the traditional absorption coefficient can be further derived based on $\alpha=1-|r|^{2}$, with $r=\left(T_{11}-Z_{0} T_{21}\right) /\left(T_{11}+Z_{0} T_{21}\right)$, where $T_{11}$ and $T_{21}$ are components of $[\mathbf{T}]$ and $Z_{0}$ is the characteristic impedance of air.

\section{The Ventilation Performance Analysis and Discussion}

The ventilation performance is verified in Figures 4(b) and 4 (c). The airflow velocity field of the "9.75-22 mm" variedunit array is displayed in Figure 4(b). The airflow can directly propagate along the gaps of the boundaries, even when the major part of the channel is blocked. In Figure 4(c), the velocity profile along the channel height $h_{5}$ is shown, and we can only see small velocity difference before and after the designed structure. Thus, it proves that our design, even for the most blocked one, can achieve high-efficiency ventilation while maintaining a broadband acoustic reduction performance in a miniaturized structure.

A ventilated broadband acoustic absorber with miniaturized metamaterial lattice array is proposed and demonstrated. The metamaterial lattice array consists of weakly coupled Archimedean spiral resonators, and different unit combinations have been analyzed and compared to achieve the optimized reduction performance. One key factor to the reduction performance of VMAs is the small coupling between different spiral units, which leads to the slight merging of resonance peaks of different modes. Another key factor is the existence of extralong inner waveguides formed by the Archimedean spiral configuration, which can improve the targeted performance and own a wide working frequency range in a relative miniaturized structure. The complex 


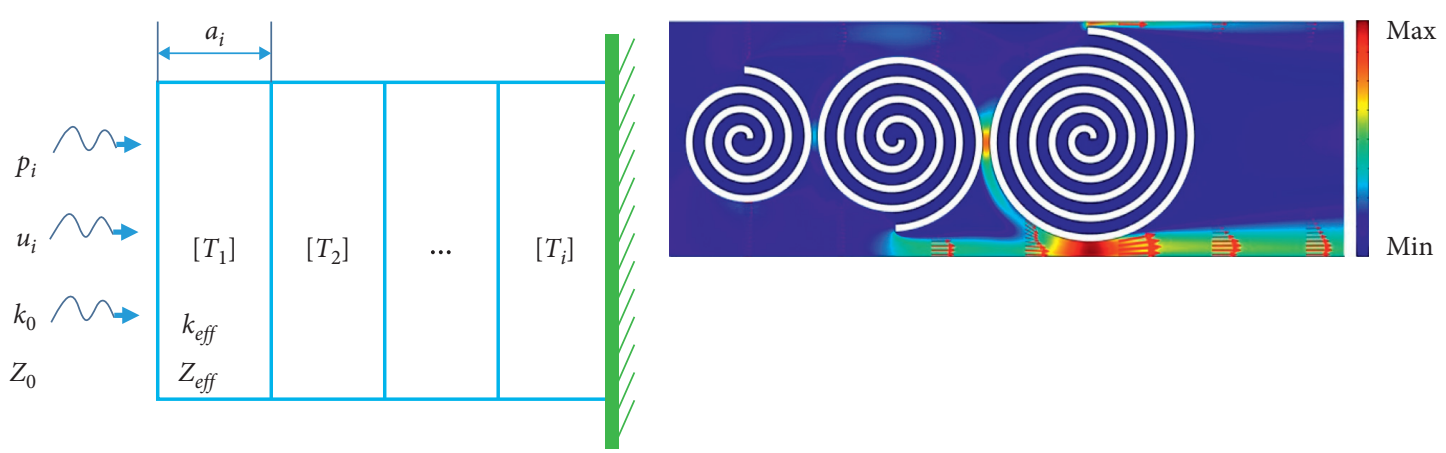

(a)

(b)

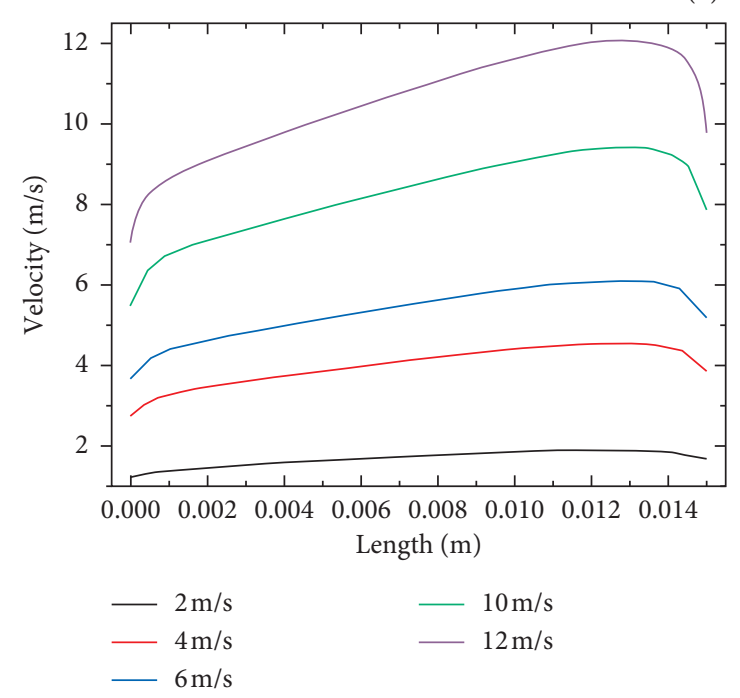

(c)

Figure 4: (a) Details of the integrated transfer matrix method. (b) The airflow velocity field of the "9.75-22 mm" varied-unit array. (c) The velocity profile along the channel height.

spiral-generated long waveguide provides a new diagram for designing effective VMAs. Moreover, the dimensional and geometric parameters can be adjusted to efficiently attenuate sounds in a broadband range while maintaining a good ventilation performance. This design overcomes the limits of previous sound absorbing structures, stepping further in practical applications for requests of both noise control and ventilation, such as flow ducts, exhaust hoods, and air conditioners, especially in application scenarios of small spaces.

\section{Data Availability}

The related data used to support the findings of this study are included within the article and Supplementary Materials.

\section{Conflicts of Interest}

The authors declare that they have no conflicts of interest.

\section{Acknowledgments}

The authors are grateful for the financial support from the National Natural Science Foundation of China (Grant nos.
51805086, 51975123, and 52005116), the Fujian Provincial Natural Science Foundation (Grant no. 2019 J01210), and Fujian Provincial Planned Projects of Joint Tackling of Health and Education (Grant no. 2019-WJ-01).

\section{Supplementary Materials}

(1) Acoustic test system and model fabrication. (2) Simulation details. (3) Pressure distributions in peak frequencies for the optimized spiral unit array. (4) The acoustic reduction performance for self-segmentation spiral unit arrays. (Supplementary Materials)

\section{References}

[1] G. Ma and P. Sheng, "Acoustic metamaterials: from local resonances to broad horizons," Science Advances, vol. 2, no. 2, Article ID e1501595, 2016.

[2] S. A. Cummer, J. Christensen, and A. Alù, "Controlling sound with acoustic metamaterials," Nature Reviews Materials, vol. 1, no. 3, pp. 1-13, 2016.

[3] H. Ge, M. Yang, C. Ma et al., "Breaking the barriers: advances in acoustic functional materials," National Science Review, vol. 5, no. 2, pp. 159-182, 2018. 
[4] M. Yang and P. Sheng, "Sound absorption structures: from porous media to acoustic metamaterials," Annual Review of Materials Research, vol. 47, no. 1, pp. 83-114, 2017.

[5] X. Wu, C. Fu, X. Li et al., "Low-frequency tunable acoustic absorber based on split tube resonators," Applied Physics Letters, vol. 109, no. 4, p. 043501, 2016.

[6] J. Li, W. Wang, Y. Xie, B.-I. Popa, and S. A. Cummer, "A sound absorbing metasurface with coupled resonators," Applied Physics Letters, vol. 109, no. 9, p. 091908, 2016.

[7] Y. Li and B. M. Assouar, "Acoustic metasurface-based perfect absorber with deep subwavelength thickness," Applied Physics Letters, vol. 108, no. 6, p. 063502, 2016.

[8] G. Ma, X. Fan, F. Ma, J. De Rosny, P. Sheng, and M. Fink, "Towards anti-causal Green's function for three-dimensional sub-diffraction focusing," Nature Physics, vol. 14, no. 6, pp. 608-612, 2018.

[9] X. Liu, Q. Guo, and J. Yang, "Miniaturization of floquet topological insulators for airborne acoustics by thermal control," Applied Physics Letters, vol. 114, no. 5, p. 054102, 2019.

[10] X. Liu, Q. Guo, and J. Yang, “The ejecting lamella of impacting compound droplets," Applied Physics Letters, vol. 115, no. 7, p. 7410, 2019.

[11] N. Sellen and M. Cuesta, "Noise reduction in a flow duct: implementation of a hybrid passive/active solution," Journal of Sound and Vibration, vol. 297, no. 3-5, pp. 492-511, 2006.

[12] P. Galland and T. Colonius, "Wave packets and turbulent jet noise," Annual Review of Fluid Mechanics, vol. 45, no. 1, pp. 173-195, 2013.

[13] D. S. Michaud, K. Feder, S. E. Keith et al., "Exposure to wind turbine noise: perceptual responses and reported health effects," The Journal of the Acoustical Society of America, vol. 139, no. 3, pp. 1443-1454, 2016.

[14] D. A. Bies, C. Hansen, and C. Howard, Engineering Noise Control, CRC Press, Boca Raton, FL, USA, 2017.

[15] M. Yang, Y. Li, C. Meng et al., "Sound absorption by subwavelength membrane structures: a geometric perspective," Comptes Rendus Mécanique, vol. 343, no. 12, pp. 635-644, 2015.

[16] C. Liu, C. Ma, X. Li, J. Luo, N. X. Fang, and Y. Lai, "Wideangle broadband nonreflecting acoustic metamaterial fence," Physical Review Applied, vol. 13, no. 5, p. 54012, 2020.

[17] X. Wu, K. Y. Au-Yeung, X. Li et al., "High-efficiency ventilated metamaterial absorber at low frequency," Applied Physics Letters, vol. 112, no. 10, Article ID 103505, 2018.

[18] V. M. García-Chocano, S. Cabrera, and J. Sánchez-Dehesa, "Broadband sound absorption by lattices of microperforated cylindrical shells," Applied Physics Letters, vol. 101, no. 18, Article ID 184101, 2012.

[19] M. Yang, C. Meng, C. Fu, Y. Li, Z. Yang, and P. Sheng, "Subwavelength total acoustic absorption with degenerate resonators," Applied Physics Letters, vol. 107, no. 10, Article ID 104104, 2015.

[20] C. Fu, X. Zhang, M. Yang, S. Xiao, and Z. Yang, "Hybrid membrane resonators for multiple frequency asymmetric absorption and reflection in large waveguide," Applied Physics Letters, vol. 110, no. 2, p. 21901, 2017.

[21] N. Jiménez, V. Romero-García, V. Pagneux, and J.-P. Groby, "Rainbow-trapping absorbers: broadband, perfect and asymmetric sound absorption by subwavelength panels for transmission problems," Scientific Reports, vol. 7, no. 1, pp. 1-12, 2017.

[22] N. Jiménez, V. Romero-García, V. Pagneux, and J.-P. Groby, "Quasi-perfect absorption by sub-wavelength acoustic panels in transmission using accumulation of resonances due to slow sound," Physical Review B, vol. 95, no. 1, p. 14205, 2017.

[23] H. Long, Y. Cheng, and X. Liu, "Asymmetric absorber with multiband and broadband for low-frequency sound," Applied Physics Letters, vol. 111, no. 14, Article ID 143502, 2017.

[24] L.-J. Li, B. Zheng, L.-M. Zhong, J. Yang, B. Liang, and J.-C. Cheng, "Broadband compact acoustic absorber with high-efficiency ventilation performance," Applied Physics Letters, vol. 113, no. 10, Article ID 103501, 2018.

[25] T. Lee, T. Nomura, E. M. Dede, and H. Iizuka, "Ultrasparse acoustic absorbers enabling fluid flow and visible-light controls," Physical Review Applied, vol. 11, no. 2, p. 24022, 2019.

[26] X. Xiang, X. Wu, X. Li et al., "Recent advances in acoustic metamaterials for simultaneous sound attenuation and air ventilation performances," Extreme Mechanics Letters, vol. 1, Article ID 100786, 2020.

[27] H.-L. Zhang, Y.-F. Zhu, B. Liang, J. Yang, J. Yang, and J.-C. Cheng, "Omnidirectional ventilated acoustic barrier," Applied Physics Letters, vol. 111, no. 20, Article ID 203502, 2017.

[28] R. Ghaffarivardavagh, J. Nikolajczyk, S. Anderson, and X. Zhang, "Ultra-open acoustic metamaterial silencer based on Fano-like interference," Physical Review B, vol. 99, no. 2, p. 24302, 2019.

[29] Y. Ge, H.-X. Sun, S.-Q. Yuan, and Y. Lai, "Switchable omnidirectional acoustic insulation through open window structures with ultrathin metasurfaces," Physical Review Materials, vol. 3, no. 6, p. 65203, 2019.

[30] X. Yu, Z. Lu, T. Liu, L. Cheng, J. Zhu, and F. Cui, "Sound transmission through a periodic acoustic metamaterial grating," Journal of Sound and Vibration, vol. 449, pp. 140156, 2019.

[31] C. Shen, Y. Xie, J. Li, S. A. Cummer, and Y. Jing, "Acoustic metacages for sound shielding with steady air flow," Journal of Applied Physics, vol. 123, no. 12, Article ID 124501, 2018.

[32] M. Sun, X. Fang, D. Mao, X. Wang, and Y. Li, "Broadband acoustic ventilation barriers," Physical Review Applied, vol. 13, no. 4, p. 44028, 2020.

[33] X. Liu, X. Cai, Q. Guo, and J. Yang, "Robust nonreciprocal acoustic propagation in a compact acoustic circulator empowered by natural convection," New Journal of Physics, vol. 21, no. 5, Article ID 053001, 2019.

[34] Z. Ruan and S. Fan, "Superscattering of light from subwavelength nanostructures," Physical Review Letters, vol. 105, no. 1, p. 13901, 2010.

[35] D. Lee and Y. Kwon, "Estimation of the absorption performance of multiple layer perforated panel systems by transfer matrix method," Journal of Sound and Vibration, vol. 278, no. 4-5, pp. 847-860, 2004.

[36] C. M. Lee and Y. Xu, "A modified transfer matrix method for prediction of transmission loss of multilayer acoustic materials," Journal of Sound and Vibration, vol. 326, no. 1-2, pp. 290-301, 2009. 